\title{
Heavy metals in vegetables and potential risk for human health
}

\author{
Fernando Guerra ${ }^{1}$, Anderson Ricardo Trevizam², Takashi Muraoka ${ }^{3 *}$, Nericlenes Chaves Marcante ${ }^{4}$, Solange Guidolin Canniatti-Brazaca ${ }^{5}$
}

IUSP/CENA - Programa de Pós-Graduação em Ciências, C.P. 96 - 13416-000 - Piracicaba, SP - Brasil.

2UNICENTRO - Depto. de Agronomia, R. Camargo Varela de Sá, 03 - 85040-080 - Guarapuava, PR - Brasil.

${ }^{3}$ USP/CENA - Lab. de Fertilidade do Solo C.P. 96 - 13416-000 - Piracicaba, SP - Brasil. 4UEMS - Programa de Pós-Graduação em Produção Vegetal, Rod. Aquidauana, km 12, C.P. 25 - 79200-000 Aquidauana, MS - Brasil.

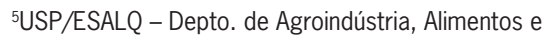
Nutrição, C.P. 09 - 13418-900 - Piracicaba, SP - Brasil.

*Corresponding author<muraoka@cena.usp.br>

Edited by: Luís Reynaldo Ferracciú Alleoni

Received November 16, 2010

Accepted June 01, 2011
ABSTRACT: Ingestion of vegetables containing heavy metals is one of the main ways in which these elements enter the human body. Once entered, heavy metals are deposited in bone and fat tissues, overlapping noble minerals. Slowly released into the body, heavy metals can cause an array of diseases. This study aimed to investigate the concentrations of cadmium, nickel, lead, cobalt and chromium in the most frequently consumed foodstuff in the São Paulo State, Brazil and to compare the heavy metal contents with the permissible limits established by the Brazilian legislation. A value of intake of heavy metals in human diets was also calculated to estimate the risk to human health. Vegetable samples were collected at the São Paulo General Warehousing and Centers Company, and the heavy metal content was determined by atomic absorption spectrophotometry. All sampled vegetables presented average concentrations of $\mathrm{Cd}$ and $\mathrm{Ni}$ lower than the permissible limits established by the Brazilian legislation. $\mathrm{Pb}$ and $\mathrm{Cr}$ exceeded the limits in $44 \%$ of the analyzed samples. The Brazilian legislation does not establish a permissible limit for Co contents. Regarding the consumption habit of the population in the São Paulo State, the daily ingestion of heavy metals was below the oral dose of reference, therefore, consumption of these vegetables can be considered safe and without risk to human health.

Keywords: contamination, food chain, potentially toxic element, daily intake, consumption habit

\section{Introduction}

Potentially harmful metal contents in soils may come not only from the bedrock itself, but also from anthropogenic sources like solid or liquid waste deposits, agricultural inputs, and fallout of industrial and urban emissions (Wilson and Pyatt, 2007). Excessive accumulation in agricultural soils may result not only in soil contamination, but has also consequences for food quality and safety. So, it is essential to monitor food quality, given that plant uptake is one of the main pathways through which heavy metals (HMs) enter the food chain (Antonious and Kochhar, 2009).

Vegetables take up HMs and accumulate them in their edible and non-edible parts at quantities high enough to cause clinical problems to both animals and human beings. As an example, the consumption of contaminated food can seriously deplete some essential nutrients in the body causing a decrease of immunological defenses, disabilities associated with malnutrition and a high prevalence of upper gastrointestinal cancer (Oliver, 1997). Lăcătuşu et al. (1996) reported that soil and vegetables contaminated with $\mathrm{Pb}$ and $\mathrm{Cd}$ in Copsa Mica and Baia Mare, Romania, significantly contributed to decrease human life expectancy (9-10 years) within the affected areas.

Cadmium and $\mathrm{Pb}$ are the most toxic elements for man (Volpe et al., 2009). In terms of environmental concentration, $\mathrm{Pb}$ is the $\mathrm{HM}$ closest to the level in which toxic signs manifest than any other substance (Baird, 2002). Others elements such as $\mathrm{Cr}$, Co and $\mathrm{Ni}$, although essential for men, at concentrations higher than those recommended, may cause metabolic disorders. Moreover, an increasing awareness in terms of the importance of vegetables and fruits to human diet suggests that the monitoring of HMs in food crops should be carried out frequently, however information concerning this issue is scarce, especially in Brazil. Most major cities have been concerned with HM contents in vegetables (FerréHuguet et al., 2008). São Paulo city, one of the largest metropolitan regions in the world, with over 39 million inhabitants, does not have this kind of monitoring and concern. Therefore, this study aimed to investigate the concentrations of $\mathrm{Cd}, \mathrm{Pb}, \mathrm{Ni}, \mathrm{Co}$ and $\mathrm{Cr}$ in the most frequently consumed vegetables of the São Paulo State, Brazil to compare then with the permissible limits established by Brazilian legislation. Also a value of intake of HMs in human diets was calculated to estimate the risk to human health.

\section{Materials and Methods}

Vegetable samples were collected in August 2009 at the São Paulo General Warehousing and Centers Company (CEAGESP), located in São Paulo/Brazil (23 $32^{\prime} 14^{\prime \prime S}$ and $46^{\circ} 44^{\prime} 14^{\prime \prime} \mathrm{W} ; 723 \mathrm{~m}$ asl), which holds the largest public warehouses of thestate and a complex of 13 central wholesalers, ensuring the supply of fruits and vegetables to the population. CEAGESP runs the Latin America's largest wholesale of fruits and vegetables, the world's third largest in volume of trade. In 2008, trades amounted 3.114 million tons of fruits, vegetables, flowers, fish and others. Samples were classified as fruits, green vegetables, leafy vegetables and general (tubers, bulbs, bean and rice), according to CEAGESP. In each group, only the most commonly traded vegetables in 2008 were collected, comprising 223 samples of 83 plant species. 
Samples were identified and packed into polyethylene bags. In the laboratory, they were washed following the same procedures as for food preparation to remove any surface deposits (Chary et al., 2008). Then, the vegetables were rewashed with deionized water and separated into edible and non-edible parts. The edible parts were air-dried at $65^{\circ} \mathrm{C}$, weighed and finely ground in a Willey mill.

A portion of $1 \mathrm{~g}$ of each sample was digested in a digestion block, with a nitric acid and perchloric acid (3:1) solution in Pyrex tubes (Miyazawa et al., 2009). After cooling they were diluted to a final volume of 25 $\mathrm{mL}$ with deionized water. All analyses were processed in triplicate. $\mathrm{HM}$ contents $(\mathrm{Cd}, \mathrm{Pb}, \mathrm{Ni}, \mathrm{Co}$ and $\mathrm{Cr})$ were determined in this solution by atomic absorption spectrophotometry (Varian-SpectrAA 140). The accuracy of the method was verified by analyzing the standard reference material 1515 - Apple Leaves (NIST).

For the total daily intake evaluation, the average metal content in each vegetable was calculated and multiplied by the respective consumption rate (IBGE, 2004). The consumption rate for children (0-6 years old) was established as $1 / 3$ of that for adults. The descriptive statistics (mean and coefficient of variation) were conducted using the Excel software.

Risk to human health by the intake of metal-contaminated vegetables was characterized using a hazard quotient (HQ) (US EPA, 1989). HQ is the ratio between exposure and the reference oral dose $\left(R_{f} D\right)$. If the ratio is lower than one (1), there will be no obvious risk. An estimate of the potential hazard to human health (HQ) through consumption of vegetables grown in metal-contaminated soil is described in Eq. (1):

$H Q=(D i v) \times\left(C_{\text {metal }}\right) / R_{f} D \times B o$

Where (Div) is the daily intake of vegetables $(\mathrm{kg}$ per day $),\left(\mathrm{C}_{\text {metal }}\right)$ is the concentration of metal in the vegetable $\left(\mathrm{mg} \mathrm{kg}^{-1}\right), \mathrm{R}_{\mathrm{f}} \mathrm{D}$ is the oral reference dose for the metal (mg kg-1 of body weight per day), and Bo is the human body mass (kg). $\mathrm{R}_{\mathrm{f}} \mathrm{D}$ is an estimate of a daily oral exposure for the human population, which does not cause deleterious effects during a lifetime, generally used in EPA's noncancer health assessments.

Values of $\mathrm{R}_{\mathrm{f}} \mathrm{D}$ for $\mathrm{Cd}\left(0.001 \mathrm{mg} \mathrm{kg}^{-1}\right.$ per day), $\mathrm{Ni}$ (0.02 $\mathrm{mg} \mathrm{kg}^{-1}$ per day) and $\mathrm{Cr}$ ( $1.5 \mathrm{mg} \mathrm{kg}^{-1}$ per day) were taken from Integrated Risk Information System (US EPA, 2010). The value of $R_{f} \mathrm{D}$ for $\mathrm{Pb}\left(0.0035 \mathrm{mg} \mathrm{kg}^{-1}\right.$ per day) was taken from WHO (1993). There is no consensus about the $\mathrm{R}_{\mathrm{f}} \mathrm{D}$ for $\mathrm{Co}$, then the oral reference dose for Co was estimated as $0.043 \mathrm{mg} \mathrm{kg}^{-1}$ per day (Food and Nutrition Board, 2004). The average Bo was taken as 70 $\mathrm{kg}$ for adults (WHO, 1993), and $19.25 \mathrm{~kg}$ for children 0-6 years old (IBGE, 2006).

To evaluate the potential risk to human health through more than one HM, the hazard index (HI) has been developed (US EPA, 1989). The hazard index is the sum of the hazard quotients, as described in Eq. (2). It assumes that the magnitude of the adverse effect will be proportional to the sum of multiple metal exposures. It also assumes similar working mechanisms that linearly affect the target organ.

$H I=\sum H Q=H Q_{C d}+H Q_{N i}+H Q_{P b}+H Q_{C o}+H Q_{C r}$

\section{Results and Discussion}

The results of the quality control are summarized in Table 1. Concentrations of $\mathrm{Cd}, \mathrm{Ni}, \mathrm{Pb}, \mathrm{Co}$ and $\mathrm{Cr}$ in vegetables (fresh weight) are shown in Tables 2, 3, 4 and 5. The concentrations of HMs in edible parts of vegetables were compared with the corresponding permissible limits established by National Agency for Sanitary Vigilance (Anvisa) (1965). According to Anvisa, the permissible limits for $\mathrm{Cd}, \mathrm{Ni}, \mathrm{Pb}$ and $\mathrm{Cr}$ are 1.0, 5.0, 0.5 and $0.1 \mathrm{mg} \mathrm{kg}^{-1}$, respectively (fresh weight), with no recommendation for Co.

The Cd concentrations ranged between 0.01 and $0.18 \mathrm{mg} \mathrm{kg}^{-1}$, and did not exceed the Brazilian limit for all vegetables. No value of $\mathrm{Ni}$, which ranged between 0.01 and $0.74 \mathrm{mg} \mathrm{kg}^{-1}$, exceeded the permissible limit. The $\mathrm{Pb}$ concentrations ranged between 0.02 and $2.50 \mathrm{mg}$ $\mathrm{kg}^{-1}$, meaning that $44.6 \%$ of the samples exceeded the permissible limit. Leafy vegetables tend to accumulate high amounts of $\mathrm{Cd}, \mathrm{Ni}$, and $\mathrm{Pb}$ due not only to their large leaf area and high transpiration rate, but also to the fast growth rate of these plants as observed by Itanna (2002). However, concentrations of $\mathrm{Pb}$, that exceeded the established permissible limit of $0.5 \mathrm{mg} \mathrm{kg}^{-1}$, were not observed for a specific class of vegetables in this study.

$\mathrm{Cr}$ concentrations ranged between 0.01 and 0.60 $\mathrm{mg} \mathrm{kg}^{-1}$ and $44.2 \%$ of the samples were found above the permissible limit, mainly for green vegetables accounting for $59.9 \%$ of the total (Table 4). The highest concentrations of $\mathrm{Pb}$ and $\mathrm{Cr}$ exceeded the permissible limits by approximately 5 (national garlic) and 6 (Japanese pumpkin) times, respectively.

It is important to observe the permissible limits for HM contents established by International Agencies for Brazilian agricultural export goods, since they could be a barrier for international trades. Besides concentrate orange juice, the main Brazilian agricultural export products in Table 2 are mango, melon, papaya, grape, lemon, apple, pineapple, banana and guava. The permissible limit established by FAO/WHO /Codex Alimentarius

Table 1 - Certified Reference Material (SRM NIST 1515) data for checking the analytical accuracy.

\begin{tabular}{|c|c|c|c|}
\hline \multirow{2}{*}{ Element } & Certified Value & Observed Value & \multirow{2}{*}{$\begin{array}{c}\text { Recover } \\
\%\end{array}$} \\
\hline & \multicolumn{2}{|c|}{$\mathrm{mg} \mathrm{kg}^{-1}$} & \\
\hline $\mathrm{Cd}$ & $0.013 \pm 0.002$ & $0.016 \pm 0.006$ & 123 \\
\hline $\mathrm{Co}^{(1)}$ & 0.09 & $0.073 \pm 0.003$ & 81 \\
\hline $\mathrm{Cr}^{(1)}$ & 0.3 & $0.225 \pm 0.048$ & 75 \\
\hline $\mathrm{Ni}$ & $0.91 \pm 0.12$ & $0.728 \pm 0.067$ & 80 \\
\hline $\mathrm{Pb}$ & $0.470 \pm 0.024$ & $0.366 \pm 0.042$ & 78 \\
\hline
\end{tabular}

(1) Noncertified value 
Table 2 - Concentrations of heavy metals in fruits (fresh weight) consumed in the São Paulo State.

\begin{tabular}{|c|c|c|c|c|c|c|c|c|c|}
\hline Vegetables & Scientific Name & $\begin{array}{l}\text { State of } \\
\text { Origin }\end{array}$ & $\mathrm{n}^{\mathrm{a}}$ & Consumption & $\mathrm{Cd}$ & $\mathrm{Ni}$ & $\mathrm{Pb}$ & Co & $\mathrm{Cr}$ \\
\hline & & & & kg per year & & 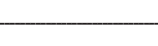 & $-\mathrm{mg} \mathrm{kg}^{-1}$ & & \\
\hline Avocado & Persea americana & $\mathrm{SP}$ & 3 & 0.34 & $0.04(0.11)^{b}$ & $0.40(0.05)$ & $0.04(0.13)$ & $0.23(0.14)$ & $0.21(0.13)$ \\
\hline 'Hawai’ Pineapple & Ananas comosus (L.) & SP & 3 & 0.44 & $0.02(0.13)$ & $0.15(0.13)$ & $0.29(0.19)$ & $0.16(0.13)$ & $0.04(0.02)$ \\
\hline ‘Pérola' Pineapple & Ananas comosus (L.) & BA & 3 & 0.44 & $0.03(0.19)$ & $0.16(0.06)$ & $0.57(0.19)$ & $0.12(0.09)$ & $0.03(0.11)$ \\
\hline ‘Nanica’ Banana & Musa ssp. & $\mathrm{SP}$ & 5 & 1.48 & $0.06(0.11)$ & $0.27(0.05)$ & $0.68(0.13)$ & $0.10(0.11)$ & $0.34(0.11)$ \\
\hline ‘Prata’ Banana & Musa sapientum Schum. & $\mathrm{SP}$ & 5 & 2.91 & $0.06(0.10)$ & $0.15(0.06)$ & $0.72(0.12)$ & $0.11(0.12)$ & $0.17(0.07)$ \\
\hline Red Guava & Psidium guajava L. & $\mathrm{SP}$ & 4 & 0.21 & $0.01(0.09)$ & $0.13(0.18)$ & $0.42(0.06)$ & $0.17(0.01)$ & $0.08(0.07)$ \\
\hline ‘Bergamota' Orange & Citrus reticulata & $\mathrm{SP}$ & 4 & 0.97 & $0.02(0.16)$ & $0.12(0.05)$ & $0.34(0.14)$ & $0.14(0.05)$ & $0.09(0.14)$ \\
\hline ‘Lima’ Orange & Citrus sinenses (L.) Osbeck & $\mathrm{SP}$ & 4 & 0.45 & $0.02(0.07)$ & $0.22(0.11)$ & $1.10(0.11)$ & $0.26(0.06)$ & $0.08(0.02)$ \\
\hline 'Mexerica' Orange & Citrus reticulata & $M G$ & 4 & 0.97 & $0.06(0.002)$ & $0.16(0.03)$ & $0.55(0.03)$ & $0.15(0.02)$ & $0.01(0.11)$ \\
\hline 'Pêra' Orange & Citrus sinenses & $\mathrm{SP}$ & 4 & 2.91 & $0.05(0.01)$ & $0.15(0.08)$ & $0.85(0.17)$ & $0.15(0.15)$ & $0.09(0.05)$ \\
\hline 'Tahiti' Lemon & Citrus aurantifolia (Christm.) Swingle & SP & 4 & 0.81 & $0.03(0.09)$ & $0.22(0.05)$ & $0.47(0.10)$ & $0.13(0.11)$ & $0.30(0.10)$ \\
\hline ‘Fuji’ Apple & Malus silvestris Mill. & SC & 3 & 0.53 & $0.02(0.16)$ & $0.05(0.11)$ & $\mathrm{nd}^{\mathrm{c}}$ & $0.08(0.17)$ & nd \\
\hline 'Gala' 1 Apple ${ }^{d}$ & Malus silvestris Mill. & SC & 3 & 0.53 & $0.03(0.07)$ & $0.04(0.16)$ & $0.29(0.09)$ & $0.06(0.10)$ & nd \\
\hline ‘Gala’ 2 Apple d & Malus silvestris Mill. & PR & 3 & 0.53 & $0.04(0.08)^{b}$ & $\mathrm{nd}^{\mathrm{c}}$ & nd & $0.02(0.15)$ & $0.02(0.13)$ \\
\hline 'Granny Smith' Apple & Malus silvestris Mill. & SC & 3 & 0.53 & $0.05(0.12)$ & $0.08(0.16)$ & $0.02(0.13)$ & $0.04(0.15)$ & $0.05(0.13)$ \\
\hline ‘Formosa’ Papaya & Carica papaya L. & ES & 3 & 1.20 & $0.02(0.09)$ & $0.05(0.10)$ & $0.39(0.14)$ & $0.08(0.15)$ & $0.02(0.07)$ \\
\hline Papaya & Carica papaya L. & $-e$ & 3 & 1.20 & $0.01(0.23)$ & $0.40(0.06)$ & $0.58(0.15)$ & $0.15(0.08)$ & $0.09(0.14)$ \\
\hline Mango & Mangifera indica L. & PE & 3 & 0.97 & $0.01(0.12)$ & $0.11(0.08)$ & $0.45(0.14)$ & $0.11(0.12)$ & $0.03(0.13)$ \\
\hline Yellow passion-fruit & Passiflora edulis f. flavicarpa & BA & 4 & 0.36 & $0.03(0.10)$ & $0.23(0.11)$ & $0.91(0.17)$ & $0.20(0.08)$ & $0.10(0.12)$ \\
\hline Watermelon & Citrullus lanatus & GO & 1 & 2.13 & 0.02 & 0.05 & 0.17 & 0.05 & 0.01 \\
\hline Melon & Cucumis melo L. & PE & 3 & 0.39 & $0.02(0.02)$ & $0.20(0.01)$ & $0.54(0.16)$ & $0.32(0.16)$ & $0.02(0.12)$ \\
\hline Strawberry & Fragaria $X$ ananassa Duch.. & SP & 5 & 0.12 & $0.03(0.04)$ & $0.10(0.08)$ & $0.40(0.05)$ & $0.14(0.13)$ & $0.04(0.01)$ \\
\hline 'Benitaca' Grape & Vitis vinifera L. & PE & 3 & 0.16 & $0.07(0.04)$ & $0.03(0.15)$ & $0.39(0.12)$ & $0.12(0.06)$ & nd \\
\hline ‘Itália’ Grape & Vitis vinifera L. & PE & 3 & 0.16 & $0.07(0.19)$ & $0.08(0.01)$ & $0.75(0.17)$ & $0.05(0.12)$ & nd \\
\hline 'Niágara' Grape & Vitis vinifera L. & $M G$ & 3 & 0.16 & $0.09(0.15)$ & $0.09(0.09)$ & $0.64(0.19)$ & $0.13(0.08)$ & $0.01(0.13)$ \\
\hline ‘Rubi’ Grape & Vitis vinifera L. & $M G$ & 3 & 0.16 & $0.09(0.14)$ & $0.08(0.11)$ & $0.49(0.18)$ & $0.11(0.13)$ & nd \\
\hline
\end{tabular}

${ }^{a} \mathrm{n}=$ number of collected samples amount. ${ }^{b}$ mean value and coefficient of variation. ${ }^{\mathrm{c}} \mathrm{nd}=$ below detection limit, $\mathrm{Ni}<0.09 \mathrm{mg} \mathrm{L}^{-1}, \mathrm{~Pb}<0.09 \mathrm{mg} \mathrm{L}^{-1}$ and $\mathrm{Cr}<0.06$ $\mathrm{mg} \mathrm{L}^{-1} \cdot{ }^{\mathrm{d}}$ apple 1 and 2 = distinct precedence. ${ }^{\mathrm{e}}$ unknown origin.

Table 3 - Concentrations of heavy metals in leafy vegetables (fresh weight) consumed in the São Paulo State.

\begin{tabular}{|c|c|c|c|c|c|c|c|c|c|}
\hline Vegetables & Scientific Name & $\begin{array}{l}\text { State of } \\
\text { Origin }\end{array}$ & $\mathrm{n}^{\mathrm{a}}$ & Consumption & $\mathrm{Cd}$ & $\mathrm{Ni}$ & $\mathrm{Pb}$ & Co & $\mathrm{Cr}$ \\
\hline \multirow[b]{2}{*}{ Chard } & \multirow[b]{2}{*}{ Beta vulgaris $\mathrm{L}$. var. cicla } & \multirow[b]{2}{*}{ SP } & \multicolumn{2}{|r|}{ kg per year } & & & $\mathrm{kg}^{-1}$ & & \\
\hline & & & 2 & 0.12 & $0.05^{b}$ & 0.21 & 0.58 & 0.17 & 0.07 \\
\hline Watercress & Nasturtium officinale sp. & SP & 2 & 0.19 & 0.10 & 0.34 & 0.86 & 0.30 & 0.34 \\
\hline Iceberg Lettuce & Lactuca sativa L. & SP & 2 & 0.22 & 0.03 & 0.13 & 0.41 & 0.07 & 0.20 \\
\hline Crisphead Lettuce & Lactuca sativa L. & SP & 2 & 0.22 & 0.08 & 0.33 & 0.48 & 0.15 & 0.20 \\
\hline Smooth Lettuce & Lactuca sativa L. & SP & 2 & 0.22 & 0.07 & 0.13 & 0.44 & 0.16 & 0.09 \\
\hline Coriander & Coriandrum sativum L. & SP & 2 & 0.02 & 0.16 & 0.57 & 1.24 & 0.50 & 0.02 \\
\hline Cabbage & Brassica oleracea var. acephala D.C. & SP & 2 & 0.09 & 0.12 & 0.54 & 1.66 & 0.51 & 0.39 \\
\hline Broccoli & Brassica oleracea $L$ & SP & 2 & 0.20 & 0.08 & 0.26 & 0.93 & 0.25 & 0.48 \\
\hline Cauliflower & Brassica oleracea var botrytis & SP & 2 & 0.29 & 0.08 & 0.24 & 0.36 & 0.15 & nd c \\
\hline Endive & Cichorium endivia L. & SP & 2 & 0.02 & 0.07 & 0.31 & 0.49 & 0.19 & 0.10 \\
\hline Spinach & Tetragonia expansa & SP & 2 & 0.02 & 0.13 & 0.48 & 1.05 & 0.46 & 0.27 \\
\hline Sweetcorn & Zea Mays L. & SP & 2 & 0.34 & 0.08 & 0.07 & 0.16 & 0.32 & nd \\
\hline White Cabbage & Brassica oleracea L. var. capitata & $-d$ & 2 & 0.50 & 0.04 & 0.21 & 0.60 & 0.23 & 0.06 \\
\hline Purple Cabbage & Brassica oleracea $L$ & - & 2 & 0.50 & 0.06 & 0.32 & 0.63 & 0.34 & 0.43 \\
\hline Rocket & Eruca sativa L. & SP & 2 & 0.02 & 0.07 & 0.29 & 0.76 & 0.21 & 0.23 \\
\hline Parsley & Petroselinum crispum (Mill.) Nym. & SP & 2 & 0.02 & 0.18 & 0.70 & 1.02 & 0.47 & 0.03 \\
\hline Celery & Apium graveolens L. & SP & 2 & 0.02 & 0.05 & 0.19 & 0.47 & 0.17 & 0.14 \\
\hline
\end{tabular}

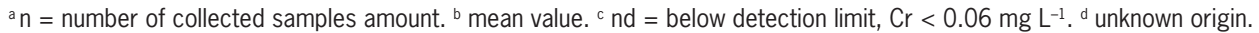


Table 4 - Concentrations of heavy metals in green vegetables (fresh weight) consumed in the São Paulo State.

\begin{tabular}{|c|c|c|c|c|c|c|c|c|c|}
\hline Vegetables & Scientific Name & $\begin{array}{c}\text { State of } \\
\text { Origin }\end{array}$ & $\mathrm{n}^{\mathrm{a}}$ & Consumption & $\mathrm{Cd}$ & $\mathrm{Ni}$ & $\mathrm{Pb}$ & Co & $\mathrm{Cr}$ \\
\hline & & & & kg per year & & - & $\mathrm{mg} \mathrm{kg}^{-1}$ & & \\
\hline 'Seca' Squash & Cucurbita moschata & $\mathrm{SP}$ & 2 & 0.25 & $0.04^{b}$ & 0.19 & 0.25 & 0.19 & 0.13 \\
\hline ‘Italian’ Pumpkin & Cucurbita pepo L. & $\mathrm{SP}$ & 2 & 0.25 & 0.04 & 0.20 & 0.30 & 0.18 & 0.08 \\
\hline ‘Japanese’ Pumpkin & Cucurbita pepo L. & $\mathrm{BA}$ & 2 & 0.25 & 0.16 & 0.24 & 0.90 & 0.62 & 0.60 \\
\hline ‘Moranga' Pumpkin & Cucurbita maxima Duch & $\mathrm{SP}$ & 2 & 0.25 & 0.06 & 0.28 & 0.56 & 0.30 & 0.13 \\
\hline ‘Paulista’ Squash & Cucurbita moschata & $\mathrm{SP}$ & 2 & 0.25 & 0.05 & 0.21 & 0.50 & 0.24 & 0.13 \\
\hline 'Italian’ Zucchini & Cucurbita pepo L. & $-\mathrm{e}$ & 2 & 0.31 & 0.04 & 0.25 & 0.51 & 0.16 & 0.08 \\
\hline ‘Paulista' Zucchini & Cucurbita moschata & $\mathrm{SP}$ & 2 & 0.31 & 0.04 & 0.23 & 0.59 & 0.19 & 0.15 \\
\hline Yellow Sweet Potato & Ipomoea batatas L. & SP & 3 & 0.13 & $0.11(0.10)$ & $0.15(0.04)$ & $0.43(0.10)$ & $0.49(0.10)$ & $\mathrm{nd}^{\mathrm{c}}$ \\
\hline White Sweet Potato & Ipomoea batatas L. & SE & 3 & 0.13 & $0.14(0.002)$ & $0.18(0.04)$ & $0.46(0.001)$ & $0.62(0.15)$ & $0.04(0.02$ \\
\hline Pink Sweet Potato & Ipomoea batatas L. & SP & 5 & 0.13 & $0.12(0.08)$ & $0.26(0.04)$ & $0.52(0.15)$ & $0.55(0.02)$ & nd \\
\hline Eggplant & Solanum melongena L. & $\mathrm{SP}$ & 2 & 0.38 & 0.04 & 0.13 & 0.44 & 0.20 & 0.16 \\
\hline Beet & Beta vulgaris $\mathrm{L}$. & $\mathrm{SP}$ & 3 & 0.44 & $0.09(0.02)$ & $0.26(0.10)$ & $0.58(0.12)$ & $0.33(0.06)$ & $0.21(0.10$ \\
\hline Yam & Dioscorea spp. & $\mathrm{SP}$ & 2 & 0.11 & 0.06 & 0.20 & 0.72 & 0.16 & 0.20 \\
\hline Carrot & Daucus corota L. & $M G$ & 3 & 2.09 & $0.03(0.01)$ & $0.11(0.12)$ & $0.38(0.09)$ & $0.15(0.05)$ & $0.09(0.10$ \\
\hline Chayote & Sechium edule Sw. & ES & 2 & 1.07 & 0.09 & 0.74 & 0.31 & 0.55 & 0.29 \\
\hline Jilo & Solanum gilo Raddi & $\mathrm{SP}$ & 2 & 0.33 & 0.06 & 0.15 & 0.28 & 0.22 & 0.26 \\
\hline Cassava $1^{\mathrm{d}}$ & Manihot esculenta Crantz. & $\mathrm{SP}$ & 2 & 0.82 & 0.13 & 0.11 & nd & 0.62 & 0.28 \\
\hline Cassava 2 & Manihot esculenta Crantz. & $\mathrm{SP}$ & 2 & 0.82 & 0.10 & 0.63 & 0.98 & 0.80 & 0.11 \\
\hline Arracacha & Arracacia xanthorrhiza Banc. & $\mathrm{SP}$ & 4 & 0.65 & $0.09(0.05)$ & $0.24(0.15)$ & $1.18(0.12)$ & $0.19(0.05)$ & $0.41(0.13$ \\
\hline 'Caipira' Cucumber & Cucumis sativus $\mathrm{L}$. & - & 2 & 0.16 & 0.02 & 0.25 & 0.30 & 0.09 & 0.05 \\
\hline ‘Comum’ Cucumber & Cucumis sativus $\mathrm{L}$. & GO & 2 & 0.16 & 0.02 & 0.16 & 0.25 & 0.08 & 0.02 \\
\hline ‘Japanese’ Cucumber & Cucumis sativus $\mathrm{L}$. & $\mathrm{SP}$ & 2 & 0.16 & 0.02 & 0.12 & 0.37 & 0.09 & 0.12 \\
\hline Green Pepper & Capsicum annuun L. & $\mathrm{SP}$ & 2 & 0.28 & 0.04 & 0.12 & 0.29 & 0.10 & 0.12 \\
\hline Red Pepper & Capsicum annuun $\mathrm{L}$. & $\mathrm{SP}$ & 2 & 0.28 & 0.05 & 0.16 & 0.25 & 0.21 & 0.20 \\
\hline Okra & Abelmoschus esculentus (L.) Moench. & SP & 5 & 0.50 & $0.08(0.12)$ & $0.48(0.03)$ & $1.31(0.07)$ & $0.36(0.05)$ & $0.21(0.06$ \\
\hline ‘Andrea' Tomato cv. & Lycopersicum esculentum Mill. & $M G$ & 2 & 1.38 & 0.03 & 0.20 & 0.17 & 0.15 & nd \\
\hline ‘Caqui’ Tomato cv. & Lycopersicum esculentum Mill. & $\mathrm{SP}$ & 2 & 1.38 & 0.04 & 0.14 & 0.20 & 0.11 & 0.01 \\
\hline ‘Long Life’ Tomato cv. & Lycopersicum esculentum Mill. & $M G$ & 2 & 1.38 & 0.03 & 0.11 & 0.21 & 0.12 & 0.04 \\
\hline ‘Rasteiro’ Tomato cv. & Lycopersicum esculentum Mill. & $\mathrm{SP}$ & 2 & 1.38 & 0.02 & 0.10 & 0.19 & 0.10 & 0.02 \\
\hline Green Bean & Phaseolus vulgaris $\mathrm{L}$. & $\mathrm{SP}$ & 4 & 0.33 & $0.07(0.06)$ & $0.19(0.13)$ & $0.75(0.11)$ & $0.30(0.08)$ & $0.15(0.11)$ \\
\hline
\end{tabular}

${ }^{a} \mathrm{n}=$ number of collected samples. ${ }^{\mathrm{b}}$ mean value and coefficient of variation. ${ }^{\mathrm{c}} \mathrm{nd}=$ below detection limit, $\mathrm{Pb}<0.09 \mathrm{mg} \mathrm{L^{-1 }}$ and $\mathrm{Cr}<0.06 \mathrm{mg} \mathrm{L}^{-1}$. ${ }^{\mathrm{d}}$ cassava 1 and $2=$ distinct precedence. ${ }^{e}$ unknown origin.

Table 5 - Concentrations of heavy metals in "general" vegetables (fresh weight) consumed in the São Paulo State.

\begin{tabular}{|c|c|c|c|c|c|c|c|c|c|}
\hline Vegetables & Scientific Name & $\begin{array}{c}\text { State of } \\
\text { Origin }\end{array}$ & $n^{\mathrm{a}}$ & Consumption & $\mathrm{Cd}$ & $\mathrm{Ni}$ & $\mathrm{Pb}$ & Co & $\mathrm{Cr}$ \\
\hline & & & & kg per year & 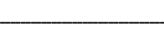 & - & $\mathrm{mg} \mathrm{kg}^{-1}$ & - & - \\
\hline Chinese Garlic & Allium sativum $\mathrm{L}$. & $-\mathrm{e}$ & 3 & 0.30 & $0.12(0.12)^{b}$ & $0.47(0.10)$ & $1.94(0.08)$ & $0.44(0.04)$ & $0.26(0.07)$ \\
\hline National Garlic & Allium sativum L. & $M G$ & 3 & 0.30 & $0.09(0.09)$ & $0.42(0.01)$ & $2.50(0.14)$ & $0.37(0.16)$ & $0.29(0.04)$ \\
\hline Rice & Oryza sativa & RS & 3 & 27.27 & nd ${ }^{c}$ & $0.20(0.11)$ & $0.23(0.14)$ & $0.18(0.16)$ & nd \\
\hline ‘Ágata’ Potato cv. & Solanum tuberosum ssp. & SP & 2 & 1.52 & 0.09 & 0.22 & 0.99 & 0.13 & 0.27 \\
\hline ‘Asterix’ Potato cv. & Solanum tuberosum ssp. & SP & 2 & 1.52 & 0.09 & 0.37 & 1.02 & 0.28 & 0.13 \\
\hline ‘Caesar' Potato cv. & Solanum tuberosum ssp. & $\mathrm{SP}$ & 2 & 1.52 & 0.11 & 0.27 & 0.48 & 0.16 & nd \\
\hline 'Monalisa' Potato cv. & Solanum tuberosum ssp. & SP & 2 & 1.52 & 0.12 & 0.22 & 0.77 & 0.21 & 0.03 \\
\hline Onion & Allium cepa L. & $M G$ & 3 & 3.47 & $0.02(0.04)$ & $0.06(0.13)$ & $0.49(0.12)$ & $0.09(0.10)$ & $0.07(0.11)$ \\
\hline Bean $1^{d}$ & Phaseolus vulgaris & - & 3 & 3.04 & $0.01(0.09)$ & $0.44(0.01)$ & $0.26(0.12)$ & $0.08(0.09)$ & $0.002(0.16)$ \\
\hline Bean 2 & Phaseolus vulgaris & - & 3 & 3.04 & $0.002(0.11)$ & $0.16(0.07)$ & $0.70(0.08)$ & $0.13(0.12)$ & nd \\
\hline
\end{tabular}


Commission, 1995) for Cd ranges between 0.05 and 0.4 $\mathrm{mg} \mathrm{kg}^{-1}$ and for $\mathrm{Pb}$ between 0.1 and $0.3 \mathrm{mg} \mathrm{kg}^{-1}$, depending on food type. The fruits previously described had $\mathrm{Cd}$ concentrations below the codex limit. $\mathrm{Pb}$ concentrations exceeded the codex limit, except for apples and 'hawai' pineapple. So, in general, Brazilian agricultural export products do not show problems on HM content. There are no reference values for other HMs in the codex.

\section{Daily intake of heavy metals}

Cadmium is a dangerous element because it can be absorbed via the alimentary tract, penetrate through placenta during pregnancy, and damage membranes and DNA. Once in the human body, it may remain in the metabolism from 16 to 33 years and is connected to several health problems, such as renal damages and abnormal urinary excretion of proteins. Decrease in bone calcium concentrations and increase of urinary excretion of calcium have also been attributed to exposure to $\mathrm{Cd}$, eventually causing death. It also affects reproduction and endocrine systems of women (WHO, 2004). Vegetables may contribute to about $70 \%$ of $\mathrm{Cd}$ intake by humans, varying according to the level of consumption (Wagner, 1993).

The daily intake of $\mathrm{Cd}$ was estimated as 0.008 $\mathrm{mg}$, which represents approximately $10.7 \%$ of the $\mathrm{R}_{\mathrm{f}} \mathrm{D}$, established to $0.001 \mathrm{mg} \mathrm{kg}^{-1}$ of body weight per day, equivalent to $0.07 \mathrm{mg}$ per day for a $70 \mathrm{~kg}$ adult ( $W H O$, 1993). The daily intake was lower than the tolerable daily intake (TDI). The content of Cd intake found was lower than that reported in literature, which ranged between 0.0018 and $0.052 \mathrm{mg}$ per day (Santos et al., 2004; Tripathi et al., 1997). The greatest contribution for Cd intake came from the 'monalisa' potato 10.00049 mg per day), followed by the 'prata' banana $(0.00046$ mg per day), 'pêra' orange (0.00041 mg per day) and carrot $(0.00032 \mathrm{mg}$ per day), accounting for $22.6 \%$ of the total daily intake.

$\mathrm{Ni}$ does not have a specific function in humans; however, it is a co-factor for some microbial intestine enzymes. Ni content in the adult human body should remain below $0.1 \mathrm{mg}$ per day, and excess may cause damages to DNA and cell structures (Kohlmeier, 2003). The daily intake of $\mathrm{Ni}$ was estimated as $0.046 \mathrm{mg}$, which represents approximately $3.3 \%$ of $\mathrm{R}_{\mathrm{f}} \mathrm{D}$ established in $0.02 \mathrm{mg} \mathrm{kg}^{-1}$ per day, equivalent to $1.4 \mathrm{mg}$ per day for a $70 \mathrm{~kg}$ adult (WHO, 1993). The daily intake was below the TDI of $1.4 \mathrm{mg}$ per day and lower than that reported in literature (0.089 and $0.231 \mathrm{mg}$ per day) (Biego et al., 1998; Santos et al., 2004). The greatest contribution for the $\mathrm{Ni}$ intake came from rice $(0.015 \mathrm{mg}$ per day) and bean 1 (0.0036 mg per day), accounting for $40.7 \%$ of the total daily intake.

The toxic effects of $\mathrm{Pb}$ focus on several organs, such as liver, kidneys, spleen and lung, causing a variety of biochemical defects. The nervous system of infants and children is particularly affected by the toxicity of this HM. Adults exposed occupationally or accidentally to excessive levels of $\mathrm{Pb}$ exhibit neuropathology. There is association between $\mathrm{Pb}$ in human body and the increase of blood pressure in adults (Maihara and Fávaro, 2006). Although $\mathrm{Pb}$ effects are more relevant for children, calculations for risk assessment were made for adults and children. The daily intake of $\mathrm{Pb}$ was estimated to 0.102 $\mathrm{mg}$, which is $41.7 \%$ of $\mathrm{R}_{\mathrm{f}} \mathrm{D}$ value of $0.245 \mathrm{mg}$ per day for a $70 \mathrm{~kg}$ adult. Although $\mathrm{Pb}$ concentrations were high in some vegetables, low consumption of vegetables results in low intake of this element. This value was below those reported in literature (0.025 and $0.521 \mathrm{mg}$ per day) (Santos et al., 2004; Tripathi et al., 1997). Rice contributed with approximately $16.84 \%$ for the $\mathrm{Pb}$ intake, followed by the 'pêra' orange $(6.68 \%)$, bean $2(5.73 \%)$, 'prata' banana (5.52\%) and onion (4.59\%).

$\mathrm{Co}$ is a constituent of the $\mathrm{B}_{12}$ vitamin. The estimated daily intake of Co was $0.040 \mathrm{mg}$, which represents $1.3 \%$ of the $\mathrm{R}_{\mathrm{f}} \mathrm{D}$ established as $3 \mathrm{mg}$ per day (Food and Nutrition Board, 2004). There are few data on Co intake. Data in the literature range between 0.012 and $1.2 \mathrm{mg}$ per day (Biego et al., 1998; Chary et al., 2008), and the Co intake estimated in this work remained below these values. Rice was the major contributor for Co intake with $0.013 \mathrm{mg}$ per day $(33.98 \%)$ followed by cassava 2 with $0.0018 \mathrm{mg}$ per day $(4.54 \%)$.

$\mathrm{Cr}$ is an important element for the insulin activity and DNA transcription. However an intake below 0.02 mg per day could reduce cellular responses to insulin (Kohlmeier, 2003). The daily intake, estimated as 0.016 $\mathrm{mg}$, was lower than the $\mathrm{R}_{\mathrm{f}} \mathrm{D}$ established at $1.5 \mathrm{mg} \mathrm{kg}^{-1}$ per day (equivalent to $105 \mathrm{mg}$ per day) (US EPA, 2010). This value was also lower than that recommended by the US National Council (NRC, 1989) for $\mathrm{Cr}^{3+}$ : from 0.05 to $0.2 \mathrm{mg}$. The daily intake of $\mathrm{Cr}$ estimated in this work was under than that reported in literature, which ranges between 0.013 and $0.098 \mathrm{mg}$ per day (Biego et al., 1998; Santos et al., 2004). The greatest contribution for $\mathrm{Cr}$ intake came from 'prata' banana (0.0014 mg per day), followed by 'nanica' banana (0.0013 mg per day) and carrot (0.0012 mg per day), accounting for $24.7 \%$ of the total daily intake.

Although the HQ-based risk assessment method does not provide a quantitative estimate for the probability of an exposed population experiencing a reverse health effect, it indeed provides an indication of the risk level due to exposure to pollutants (Chary et al., 2008). Many researchers consider the risk estimation method reliable (Chary et al., 2008; Khan et al., 2008; Wang et al., 2005) and it has been proven to be valid and useful. However, this HQ method considers only exposure to HMs via consumption of vegetables, without taking into account other vias like dermal contact, soil ingestion, and other factors such as the presence of agrochemicals and herbicide molecules.

HQs for individual vegetables were all below 1.0 for all $\mathrm{HMs}$, regardless the population type. HQ for $\mathrm{Cd}$, $\mathrm{Ni}, \mathrm{Pb}$ and Co ranged from 0 to 0.0071 ; from 0 to 0.0107 ; from 0 to 0.0701 and from 0 to 0.0045 for adults and 
Table 6 - Values of Hazard Quotients (HQ) and Hazard Index (HI).

\begin{tabular}{lcccccc}
\hline Population & $\mathrm{Cd}$ & $\mathrm{Ni}$ & $\mathrm{Pb}$ & $\mathrm{Co}$ & $\mathrm{Cr}$ & $\mathrm{Hl}$ \\
Adults & 0.107 & 0.033 & 0.417 & 0.013 & 0.00015 & 0.57 \\
Children & 0.130 & 0.039 & 0.499 & 0.016 & 0.00018 & 0.68
\end{tabular}

from 0 to 0.0075 ; from 0 to 0.0114 ; from 0 to 0.0747 and from 0 to 0.0048 for children. Cr values were lower than 0.00001 for children and adults. When the consumption habit of adults and children in the São Paulo State were analyzed, HQs for all HMs were below 1.0 (Table 6). So, the consumption of these vegetables can be considered safe with no risk to human health.

The sequence of HQ for adults and children followed the decrescent order $\mathrm{Pb}>\mathrm{Cd}>\mathrm{Ni}>\mathrm{Co}>\mathrm{Cr}$ (Table 6). The sequence was the same for both; however the HQ for children was higher than that for adults, as verified by other researchers (Huang et al., 2008).

High $\mathrm{Pb}$ concentrations observed in many vegetables, although they do not pose a risk to human health, may be attributed to crops located near roads of heavy traffic. The main sources of this element to humans are inhalation of airborne $\mathrm{Pb}$ from vehicle emissions and from direct atmospheric deposition on soils, water, and crops, constituting the gateway into the food chain (Baird, 2002).

When the hazard index exceeds 1.0 , there is concern for potential health effects (Huang et al., 2008). Even though there was no apparent risk when each metal was analyzed individually, the potential risk could be multiplied when considering all HMs. HI for adults and children were 0.57 and 0.68 , respectively (Table 6). Although HI was higher for children, neither population suffered from ingestion of vegetables contaminated with HMs.

The relative contributions of $\mathrm{Cd}, \mathrm{Ni}, \mathrm{Pb}$ and $\mathrm{Co}$ to the aggregated risk were $18.8 \%, 5.7 \%, 73.1 \%$ and $2.3 \%$ for adults and $19.1 \%, 5.7 \%, 73.4 \%$ and $2.4 \%$ for children, respectively. $\mathrm{Cr}$ content was minimal, only $0.03 \%$, which may be related to the high $\mathrm{R}_{\mathrm{f}} \mathrm{D}$ of $\mathrm{Cr}(1.5$ mg kg-1 per day). Huang et al. (2008), and Wang et al. (2005) also found minimal contribution of $\mathrm{Cr}$ to aggregated risks via consumption of wheat grains and vegetables in Kunshan and Tianjin, China.

\section{Acknowledgements}

To FAPESP for the financial support, Rodrigo Coqui da Silva and Diogo Néia Eberhardt for their constructive criticism, which has improved the final version of this manuscript.

\section{References}

Antonious, G.; Kochhar, T. 2009. Mobility of heavy metals from soil into hot pepper fruits: a field study. Bulletin of Environmental Contamination and Toxicology_82: 59-63.

Baird, C. 2002. Environmental Chemistry. Bookman, Porto Alegre, RS, Brazil (in Portuguese).
Biego, G.H.; Joyeux, M.; Hartemann, P.; Debry, G. 1998. Daily intake of essential minerals and metallic micropollutants from foods in France. Science of the Total Environment 217: 27-36.

Brazilian Institute of Geography and Statistics [IBGE]. 2004. Per Capita Domicile Food Acquisition: Familiar Budget Survey; 2002-2003. IBGE, Rio de Janeiro, RJ, Brazil (in Portuguese).

Brazilian Institute of Geography and Statistics [IBGE]. 2006. Anthropometry and Children and Teenager Nutritional State Analysis in Brazil: Familiar Budget Survey; 2002-2003. IBGE, Rio de Janeiro, RJ, Brazil (in Portuguese).

Chary, N.S.; Kamala, C.T.; Raj, D.S.S. 2008. Assessing risk of heavy metals from consuming food grown on sewage irrigated soils and food chain transfer. Ecotoxicology and Environmental Safety 69: 513-24.

Codex Alimentarius Commission. 1995. Codex general standard for contaminants and toxins in food and feed. Available at: http://www.codexalimentarius.net/download/standards/17/ CXS_193e.pdf_[Accessed Mar. 10, 2010].

Ferré-Huguet, N.; Marti-Cid, R.; Schuhmacher, M.; Domingo, J.L. 2008. Risk assessment of metals from consuming vegetables, fruits and rice grown on soils irrigated with waters of the Ebro River in Catalonia, Spain. Biological Trace Element Research 123: 66-79.

Food and Nutrition Board. 2004. Dietary Reference Intakes [DRIs]: Recommended Intakes for Individuals. National Academy of Sciences, Washington, DC, USA.

Huang, M.L.; Zhou, S.L.; Sun, B.; Zhao, Q.G. 2008. Heavy metals in wheat grain: Assessment of potential health risk for inhabitants in Kunshan, China. Science of the Total Environment 405: 54-61

Itanna, F. 2002. Metals in leafy vegetables grown in Addis Ababa and toxicological implications. Ethiopian Journal of Health Development 6: 295-302.

Khan, S.; Cao, Q.; Zheng, Y.M.; Huang, Y.Z.; Zhu, Y.G. 2008. Health risks of heavy metals in contaminated soils and food crops irrigated with wastewater in Beijing, China. Environmental Pollution 152: 686-692.

Kohlmeier, M. 2003. Nutrient Metabolism. Elsevier, San Diego, CA, USA.

Lăcătușu, R.; Rauta, C.; Carstea, S.; Ghelase, I. 1996. Soil-plantman relationships in heavy metal polluted areas in Romania. Applied Geochemistry 11: 105-107.

Maihara, V.A.; Fávaro, D.I.T. 2006. Toxic Elements. p. 629-660. In: Cozzolino, S.M.F., ed. Bioavailability of nutrients. Manole, Barueri, SP, Brazil (in Portuguese).

Miyazawa, M.; Pavan, M.A.; Muraoka, T.; Carmo, C.A.F.S.; Melo, W.J. 2009. Chemical Analysis of Plant Tissues. p. 191233. In: Silva, F.C., ed. Manual of chemical analysis of soils, plants and fertilizers. Embrapa Informação Tecnológica, Brasília, DF, Brazil (in Portuguese).

National Agency for Sanitary Vigilance [Anvisa]. 1965. Decree ${ }^{\circ}$ 55.871. Available at: http://www.anvisa.gov.br/legis/decretos/55871_65.htm_[Accessed Mar. 16, 2010]. (in Portuguese).

National Research Council [NRC]. 1989. Recommended Dietary Allowances. National Academy Press, Washington, DC, USA.

Oliver, M.A. 1997. Soil and human health: a review. European Journal of Soil Science 48: 573-592. 
Santos, E.E.; Lauria, D.C.; Porto da Silveira, C.L. 2004. Assessment of daily intake of trace elements due to consumption of foodstuffs by adult inhabitants of Rio de Janeiro city. Science of the Total Environment 327: 69-79.

Tripathi, R.M.; Raghunath, R.; Krishnamoorthy, T.M. 1997. Dietary intake of heavy metals in Bombay city, India. Science of the Total Environment 208: 149-159.

U.S. Environmental Protection Agency [US EPA]. 1989. Risk Assessment Guidance for Superfund: Human Health Evaluation Manual [Part A]: Interim Final. U.S. Environmental Protection Agency, Washington, DC, USA [EPA/540/1-89/002].

U.S. Environmental Protection Agency [US EPA]. 2010. Integrated risk information system. Available at: http://cfpub.epa.gov/ ncea/iris/compare.cfm [Accessed Jan. 08, 2010].

Volpe, M.G.; La Cara, F.; Volpe, F.; De Mattia, A.; Serino, V.; Pettito, F.; Zavalloni, C.; Limone, F.; Pellecchia, R.; De Prisco, P.P.; Di Stasio, M. 2009. Heavy metal uptake in the enological food chain. Food Chemistry 117: 553-560.

Wagner, G.J. 1993. Accumulation of cadmium in crop plants and its consequences to human health. Advances in Agronomy 51: 173-212.
Wang, X.L.; Sato, T.; Xing, B.S.; Tao, S. 2005. Health risks of heavy metals to the general public in Tianjin, China via consumption of vegetables and fish. Science of the Total Environment 350: 28-37.

Wilson, B.; Pyatt, F.B. 2007. Heavy metal dispersion, persistence, and bioaccumulation around an ancient copper mine situated in Anglesey, UK. Ecotoxicology and Environmental Safety 66: 224-231.

World Health Organization [WHO]. 1993. Evaluation of Certain Food Additives and Contaminants. In: Forty-First Report of the Joint FAO/WHO Expert Committee on Food Additives., WHO, Geneva, Switzerland. (WHO Technical Series, 837).

World Health Organization [WHO]. 2004. Evaluation of certain food additives and Contaminants. In: Sixty-First Report of the Joint FAO/WHO Expert Committee on Food Additives. WHO, Geneva, Switzerland. (WHO Technical Series, 922). 